Article

\title{
Responder Feelings in a Three-Player Three-Option Ultimatum Game: Affective Determinants of Rejection Behavior
}

\author{
Hans-Rüdiger Pfister ${ }^{1, *}$ and Gisela Böhm ${ }^{2}$ \\ 1 Leuphana University Lüneburg, Wilschenbrucher Weg 84, D-21335 Lüneburg, Germany; \\ E-Mail: pfister@uni-lueneburg.de \\ 2 University of Bergen, Christiesgt. 12, N-5015 Bergen, Norway; \\ E-Mail: gisela.boehm@psysp.uib.no \\ * Author to whom correspondence should be addressed; E-Mail: pfister@uni-lueneburg.de; \\ Tel.: +49-4131-677-7759; Fax: +49-4131-677-7935.
}

Received: 24 October 2011; in revised form: 16 January 2012 / Accepted: 7 February 2012 / Published: 13 February 2012

\begin{abstract}
This paper addresses the role of affect and emotions in shaping the behavior of responders in the ultimatum game. A huge amount of research shows that players do not behave in an economically rational way in the ultimatum game, and emotional mechanisms have been proposed as a possible explanation. In particular, feelings of fairness, anger and envy are likely candidates as affective determinants. We introduce a three-player ultimatum game with three-options, which permits the responder to either penalize the proposer or to penalize a third party by rejecting offers. This allows for partially distinguishing rejections due to a retaliation motive driven by anger towards the proposer from rejections due to inequity aversion driven by feelings of envy towards a third party. Results from two experiments suggest that responders experience feelings of dissatisfaction and unfairness if their share is small in comparison to the proposer's share; anger, then, may trigger rejections towards the proposer. Responders also experience dissatisfaction and envy when third party shares exceed their own shares; however, in contrast to anger, envy does not trigger rejections and is dissociated from the decision to accept or reject an offer. We conclude that acting upon anger is socially acceptable, whereas envy is not acceptable as a reason for action. Furthermore, we find that responders generally feel better after rejections, suggesting that rejections serve to regulate one's affective state.
\end{abstract}

Keywords: ultimatum game; emotions; fairness; satisfaction; anger; envy 


\section{Introduction}

Ample evidence shows that in strategic situations actual behavior is often inconsistent with optimal solutions according to standard rational choice theory [1-4]. In the well-known ultimatum game, players are willing to forego monetary gains in order to punish unfair behavior of others [4,5]. We assume that affect and emotions constitute proximal causes of behavior in such strategic games. Recent work on affective processes in decision-making demonstrates that affect and emotions frequently play an important role in shaping individuals' choices [6-9]. Drawing on findings from behavioral and psychological research on strategic interactions [10-14], we aim to contribute to this line of research and to examine the role of affect in the ultimatum game from the perspective of the responder. We study the relative impact of two responder-related motivations assumed to be emotionally triggered: One is the propensity to reject in order to punish another person because of his or her malicious intentions, a second propensity is to reject due to a general aversion towards unequal payoffs among involved parties [15]. We further presume that in either case rejections serve to regulate the responder's emotions, that is, to readjust to an emotionally acceptable state after having experienced an unpleasant offer [16].

In the original ultimatum game [4,5], a monetary endowment is given to Player 1, the proposer. The proposer is then to offer a share to Player 2, the responder. The proposer is free to offer any amount, from a minimum share of one monetary unit up to the entire endowment. The responder may accept or reject the offer. If the responder accepts, both players receive their respective shares. If the responder rejects, both players are left with nothing. Assuming that people are purely selfish and only interested in maximizing monetary gains, and that this is common knowledge, the rational solution is for the proposer to offer only the minimum amount, and for the responder to accept it, because some money is better than none. Empirically, however, proposers commonly allocate a substantial share to the responder; frequently, they offer an even 50:50 split [17]. Similarly, responders usually reject offers that they consider to be too small, thereby incurring a factual monetary loss. Offers of less than about $30 \%$ of the endowment are generally rejected. Although there is considerable variation across cultures, the economically rational behavior is extremely rare $[18,19]$.

In the present paper, we examine the mediating role of affective states in determining the choices of responders. Specifically, we examine if and when rejections are driven either by negative feelings triggered by unequal shares, or by negative feelings triggered by unfair intentions of others. A new version of a three-player, three-option ultimatum game is introduced, which allows for partially disentangling these conflicting motivations. By introducing a third party and two different types of rejection (in addition to the option of accepting, rejections can be targeted at the proposer or at the third party), it becomes possible to test whether rejections are primarily aimed at punishing the proposer, or at preventing the third party from obtaining inequitably large shares, or both.

We report two experimental studies. In both of them, it turned out that responders' rejections are almost exclusively targeted at the proposer, and are driven by judgments of fairness and by feelings of anger. Responders feel anger towards proposers deemed malicious, and anger mediates rejections that punish the proposer at a cost for the responder.

Furthermore, rejections tend to re-establish positive feelings, and thus serve a regulating function. From a purely monetary perspective, rejections are irrational. From an emotional perspective, 
however, rejections can have positive consequences in that they reduce unpleasant feelings elicited by unfair offers.

We also find that in addition to anger, responders also experience envy in response to uneven shares. Responders are dissatisfied and feel envy if third parties obtain a larger share than they do themselves; but these feelings are not enacted in resentful behavior towards the third party. Apparently, some emotions such as envy are elicited by unequal and unfair shares, but not enacted in the context of strategic interactions. This novel finding sheds some light on the controversy on intention-based versus inequity-based rejections, and emphasizes the importance of examining the role of specific emotions in strategic games.

\section{Reciprocity, Equality and Emotions}

Strategic interactions can trigger a rich array of affective responses, and individual choice behavior-be it selfish or cooperative - is frequently associated with affective responses [9,13,14,20-23]. Social emotions, as conceptualized in appraisal theories [24], such as anger, shame, guilt or envy, may play a particularly important role [25-27]. To forego a monetary gain might be compensated for by an emotional gain, for example, satisfaction from punishing a greedy proposer. Likewise, to accept an unfair monetary offer might incur emotional costs, for example, feelings of regret and self-reproach. Presumably, the actual choice will emerge from a trade-off between monetary gains and losses on the one hand, and emotional gains and losses on the other hand $[9,28]$.

\subsection{Distributional Equality and Reciprocity}

Behavior in the ultimatum game, in particular the behavior of the responder, has for the most part been attributed either to concerns about the equality of the allocated distribution of monetary shares $[29,30]$, or to concerns about reciprocity and retaliation, or both [31,32]. So far, empirical evidence is still inconclusive about the relative merits of these two explanations [15,33-36]. It should be noted that there is a related and sizable literature on distributive and procedural justice, in particular the important work by Tyler [37,38], emphasizing the significance of interpersonal aspects of procedures in establishing a feeling of justice. However, a discussion of these approaches is beyond the scope of this paper, where we focus on approaches more commonly linked to highly impoverished situations such as the ultimatum game.

The ERC (Equity, Reciprocity, Competition) model proposed in a seminal work by Bolton and Ockenfels [29] emphasizes responders' concerns about distributional equality; Fehr and Schmidt [30] argue along similar lines. The ERC model states that behavior in the ultimatum game is determined by a motivation function which includes a player's absolute payoff as well as his or her relative payoffs in comparison to what other players obtained. The basic assumption is that individuals are inherently inequality-averse, that is, they prefer monetary distributions that are equal across players. The ERC model as well as the Fehr and Schmidt model are compatible with a range of empirical findings. The models are purely distributional, assuming that players care only about their own payoffs and about the distribution of payoffs. Players worry neither about how the distribution came about nor about which intentions other players may have had when they decided on a specific split. From this model's 
perspective, an emotion such as envy, arising from an unfavorable distribution, might play an important role as the proximate cause of a responder's rejection.

In a different line of research, beginning with a contribution by Rabin [32], it is assumed that players take beliefs about the intentions of other players into account when considering which strategy to play. In particular, if a player believes that the other player is intentionally helping her, she will also be motivated to help him. Otherwise, if she believes that he is intentionally hurting her, she will be motivated to punish him, even if she has to sacrifice some or all of her own monetary gains. If both players' considerations involve a strong emotion, say hostility, they might lock in on what Rabin calls a fairness equilibrium (or hostility equilibrium, respectively): Hostile punishments of the other then constitute mutual best responses.

Dickinson [35] found supporting evidence for Rabin's model; and further evidence from public goods experiments indicates that individuals will punish others when they consider them to have acted in an unfair manner, even if punishment is costly and no immediate reciprocal benefit is to be expected [25,39]. Fehr and Gächter [25] called this phenomenon altruistic punishment, and found preliminary evidence that it might be mediated by feelings of anger. It is plausible to assume that beliefs about others' intentions elicit feelings such as anger, hostility, gratefulness or liking as proximate causes of the decision what strategy to play.

In sum, beliefs about others' intentions can be assumed to influence strategic decisions, and an emotion such as anger might be a critical determinant. Note, however, that these economically motivated models [29,30,32] all rely on a unitary utility function based solely on particular modifications of the monetary payoff distribution; such models do not incorporate emotions as particular mental states.

\subsection{Emotions in Strategic Interactions}

Our view of emotions is based on the assumption that specific emotions are mental construals elicited by cognitive appraisals of particular events, situations or agents, and are based on a general valenced affective response [24,40,41]. Furthermore, specific emotions typically motivate specific behavioral tendencies [42-44]. For example, anxiety elicits an impulse to withdraw, regret to undo a regrettable action, and envy to harm the envied person. In particular, moral and social emotions such as shame, guilt, anger, or gratitude can exert a strong influence on behavior in strategic interactions $[9,21,45,46]$.

We assume that general feelings of satisfaction and dissatisfaction as well as specific emotions can play a role when responders decide to accept or reject the offer of the proposer. While this idea has been expressed before in the game theory literature [32,45,47], relatively little direct experimental work has been done along these lines. A mounting literature confirms the importance of general affect as well as of specific emotions in individual decision-making [7-9,48-51], but only few studies have examined the preconditions and psychological mechanisms of affect and emotions in strategic games.

With respect to general affect, Kirchsteiger, Rigotti, and Rustichini [52] induced states of good or bad mood and found that bad mood leads to relatively more reciprocity, whereas good mood leads to more generosity of second movers in a gift exchange game. For the ultimatum game, this suggests that rejections might be more likely when responders are in a negative mood, implying a propensity to punish and retaliate. 
With respect to specific emotions, we assume that anger and envy are particularly relevant in these contexts. According to Ortony, Clore, and Collins [24], anger is elicited if one appraises an action of another person as violating a social norm, and if that action has negative consequences for oneself or closely related others. Anger is arguably one of the best studied social emotions, playing a key role in situations of social conflict and dispute [53]. Van Kleef et al. [53] review evidence that anger has intrapersonal effects, affecting an individual's cognitions and behavior, as well as interpersonal effects, signaling to others an individual's intentions and commitments. For example, if negotiators perceive their opponent to be angry, they tend to make larger concessions than when the opponent is perceived to be happy. In the ultimatum game, anger should be triggered by intentional violations of a social fairness norm, that is, by an offer which is substantially below an even split. Bosman et al. [27] and Reuben and van Winden [54] present evidence suggesting that responders' specific expectations that a social norm is met by another person are particularly important in the emergence of anger.

In contrast to anger, envy refers to what another person possesses in comparison to one's own endowments, rather than to what another person does. Envy is based on social comparison processes [55], and is triggered when someone believes that he or she compares unfavorably to somebody else with respect to a desired possession or self-relevant attribute. Note that envy may emerge even if the envied person does not play any active or intentional role. Envy is especially strong if the other person is perceived as similar to oneself, and if the other's advantage is judged to be undeserved [55,56]. Similarly to anger, envy motivates hostile behaviors towards the envied person, primarily to destroy the other's advantageous possession, even at a cost for oneself [57,58]. Strategic games typically lead to an unequal distribution of payoffs among the players, creating the precondition for envy to arise.

Envy has barely been studied in the context of strategic games. Kirchsteiger [26] proposed a theoretical model incorporating envy as a formal component of the proposer's utility function. He interprets the proposer's behavior as driven not by fairness concerns but by fear of the responder's envious response; however, we know of no empirical test of this model.

Empirical studies investigating emotions in strategic interactions and, in particular, in the ultimatum game, have almost exclusively dealt with anger. In a pioneering study, Pillutla and Murnighan [12] proposed a wounded pride/spite model predicting that responders feel anger after detecting unfair offers, and will then react spitefully, that is, reject the offer. Their findings suggest that the opportunity to infer unfair behavior is an important determinant of rejections, and that it is not the perception of unfairness per se, but the degree of anger felt by the responder that motivates rejections in the ultimatum game.

Srivastava et al. [14] for the first time provided empirical evidence confirming the mediating role of anger when responders retaliate against unequal offers in a traditional two-player ultimatum game. Two aspects from their study are noteworthy, namely, the direct assessment of anger via ratings, and the successful decoupling of anger and unfairness via an external attribution manipulation. Their findings strongly suggest that low offers in fact elicit anger, that anger mediates rejections, and that rejections decrease if participants are led to believe that their anger is caused by an unrelated external event. By and large, our findings on anger in Study 2 corroborate Srivastava et al.'s conclusions.

In a similar vein, a study by Bosman and van Winden [20] indicates that high emotional intensity in a power-to-take game can drive participants to destroy all of their own monetary endowments. 
In particular, intense feelings of irritation and contempt are related to self-destructive behavior, a pattern Bosman and van Winden [20] call emotional hazard.

Concerning the interpersonal function of anger, van Kleef et al. [53] report studies investigating the effect of the responder's signaling anger to the proposer. Their findings suggest that on the one hand, communicating anger may increase the offers in the ultimatum game. On the other hand, if stakes are low and if the proposer is able to deceive the responder, the responder's anger may backfire, that is, the proposer may offer even lower shares.

\subsection{Regulation of Negative Emotions}

When people experience negative emotions, an immediate response is often to try to reestablish a more positive feeling, or to attenuate the intensity of negative feelings. This process of emotion regulation [59] may comprise one or more of several strategies, such as a reappraisal of the situation, a self-serving attribution of causes, or a direct modification of the situation, and can be automatic or intentional. We propose that to reject in the ultimatum game is also a suitable strategy to restore a less aversive affective state after one has been treated in an unfair manner. In particular, the satisfaction the responder derives from a retaliating rejection might well compensate the offense experienced from a malicious offer, and leave the responder in a less unpleasant state.

\section{A Three-Player Three-Option Ultimatum Game}

We conducted two studies based on an extended version of the ultimatum game, with three players and three options for the responder. A number of studies have used versions of three-player games $[18,33,36,60,61]$. The version we implement here is novel in that it additionally provides three options to respond, which were designed to disentangle responses towards the proposer from responses towards a third party.

In the traditional two-player game, a proposer is provided with an original endowment $x$. The proposer decides on a split of the endowment among him- or herself and a second individual, the responder, so that the responder is offered a share of size $s$, leaving the remaining share of $x$ minus $s$ to the proposer. The responder decides whether to accept or reject the proposer's offer $(x-s, s)$. Upon acceptance, the responder receives $s$, and the proposer receives $x-s$. Upon rejection, both receive nothing. In the three-player version used here, the proposer decides how to share the endowment $x$ among three players: a share $p$ for the proposer, a share $s$ for the so called active responder, and a share $t=x-p-s$ for a second (inactive) responder, called the third party. With a minimum share of one monetary unit for each player, the proposer is free to offer any distribution $(p, s, t)$, with $p+s+t=x$ (and all shares $\geq 1$ unit). Full information is provided about the offer $(p, s, t)$ to the responder, and no special outside payoffs for any of the players are involved.

Furthermore, our three-option version of the ultimatum game provides three response options to the active responder. First, the responder can accept the proposal, whereupon each player obtains the offered share $(p, s, t)$. Alternatively, the responder can reject the offer in one of two ways. A rejection of Type 1 (proposer-directed rejection) implies that neither the responder nor the proposer gets anything, but the third party keeps his or her share, yielding the payoff vector $(0,0, t)$. A rejection of Type 2 (third party-directed rejection) implies that neither the responder nor the third party gets anything, but 
the proposer keeps her or his share, yielding the payoff vector $(p, 0,0)$; of course, whatever rejection the active responder chooses, his or her own payoff $s$ is foregone.

\section{Hypotheses}

First, we want to clarify the relative impact of retributive reciprocity and aversion towards unequal shares. Thus, the amount offered to the active responder, and the discrepancy of offers between the active responder and the third party will be manipulated as independent variables. We hypothesize that people will show a propensity to attribute unfair offers to the proposer, as the proposer is the active agent, and if they choose to reject at all will favor rejections of Type 1 (proposer-directed). However, we hypothesize that enlarging the discrepancy in shares between the active responder and the third party will also increase the occurrence of Type 2 (third party-directed) rejections due to an increasing salience of distributional inequality.

Second, we hypothesize that decisions to reject are mediated by judgments of fairness and by negative emotions. When unpleasant feelings are absent, responders are assumed to adopt a more economically rational approach and accept low offers. When negative feelings become more intense, however, the tendency to reject is expected to increase. That is, we assume that offers considered as unfair will tend to elicit negative feelings, which in turn will motivate responders to reject. Specifically, we hypothesize that low offers will generally trigger negative feelings towards the proposer, and that unequal shares between the responder and the third party will trigger negative feelings towards the third party. We hypothesize that anger mediates rejections towards the proposer, and envy mediates rejections towards the third party.

Third, we hypothesize that responders will generally feel better after rejections, in particular after rejections triggered by unfair offers. Irrespective of the target of rejection (proposer, third party), it is assumed that due to their regulative function, rejections ameliorate the affective state of the responder.

\section{Study One}

\subsection{Participants}

A sample of 127 undergraduate students from various study programs participated in partial fulfillment of a course requirement.

\subsection{Design}

Six ultimatum game scenarios following the structure of the three-player, three-option game as outlined above were constructed. Participants were assigned to the role of the active responder, and were informed about different offers of shares proposed by a proposer who was endowed with 100 Euros. The share offered to the participant was varied to be either 1 or 20 Euros (responder share), and the share offered to the third party was varied to be 1, 20, or 40 Euros (third party share). By implication, the proposer's share was 100 minus the responder's share minus the third party's share. For example, an offer consisting of 20 Euros for the responder and 40 Euros for the third party left the proposer with a share of 40 Euros. Note that this introduces a dependency between shares, for example, varying third party share and keeping responder share constant, leads to a corresponding 
variation of proposer share. By keeping the proposer's share substantially above the other players' shares (except in the 40, 20, 40 condition), we attempt to focus participants' attention on the relative differences between their share and the third party's share, with the proposer share being encoded as persistently much larger. The combination of responder share levels and third party share levels yields six experimental conditions in a 2 (responder share: 1 vs. 20) $\times 3$ (third party share: 1 vs. 20 vs. 40) design with repeated measurement on both independent variables.

\subsection{Material and Procedure}

All instructions and tasks were included in a questionnaire. Participants were instructed to adopt the role of the active responder and worked on all six responder scenarios, yielding a full repeated measurement design. The sequence of scenarios was randomized across participants. All choices were hypothetical; that is, participants were instructed to imagine the situation as if it were real, and to choose accordingly. There was no time restriction for filling in the questionnaire. No real monetary payoffs were paid.

The validity of this type of hypothetical task without real monetary incentives has been doubted, as it requires that participants are able to imagine the situation and to behave as if it were real. In experimental economics, only experiments with real monetary incentives are usually considered as valid [62]. However, empirical evidence suggests that in many typical cases the difference between hypothetical and incentivized experiments is small to negligible, and participants seem to be able and willing to indicate how they would decide if the consequences were real [63,64]. This allows the implementation of repeated measurement designs at low cost. Still, it is not guaranteed for each particular experimental scenario that a hypothetical design is valid. In study two we provide real monetary incentives, and discuss the contentious issue of hypothetical scenarios in the general discussion.

Participants (=responder) first read a cover story about a Mister Weber (=proposer) who had received the endowment, and who had then decided on an offer to the participant and a hypothetical third person, called Mister Paulsen (=third party). After reading the scenario, participants indicated their choice by selecting one of three options: (1) I accept the offer and everybody receives his or her proposed share; (2) I reject, and I and Mister Paulsen get nothing, but Mister Weber will keep his share; (3) I reject, and I and Mister Weber get nothing, but Mister Paulsen will keep his share. After having made their decision, participants rated the perceived fairness of the offer $(0=$ very unfair, $7=$ very fair $)$ as well as their overall feeling of satisfaction $(0=$ very dissatisfied, $7=$ very satisfied $)$ on 8 -point rating scales.

\subsection{Results}

Acceptances and rejections. First, we examined if rejections depend on the distribution of shares that were allocated to the three parties. Across all conditions, $46.1 \%$ of the offers were accepted; $50.1 \%$ were rejected together with the proposer share (Type 1 rejections), and only $3.8 \%$ were rejected together with the third party share (Type 2 rejections; see Tables 1 and 2). Third party rejections varied with responder share (Table 1): There were twenty rejections when responder share was 1 Euro, and only nine rejections when responder share was 20 Euros. 
Table 1. Number and percentage of choices by responder share (Study 1; Percentages in columns ' 1 ' and ' 20 ' are row percentages per responder share; percentages in column 'Sum' are column percentages across choice options).

\begin{tabular}{|c|c|c|c|}
\hline \multirow{2}{*}{ Choice Option } & \multicolumn{2}{|c|}{ Responder Share } & \multirow{2}{*}{ Sum } \\
\hline & 1 & 20 & \\
\hline Accept & $61(17.4 \%)$ & $290(82.6 \%)$ & $351(46.1 \%)$ \\
\hline Proposer-Reject & $300(78.5 \%)$ & $82(21.5 \%)$ & $382(50.1 \%)$ \\
\hline Third-Party-Reject & $20(69.0 \%)$ & $9(31.0 \%)$ & $29(3.8 \%)$ \\
\hline
\end{tabular}

Table 2. Number and percentage of choices by third party share (Study 1; Percentages in columns ' 1 ', ' 20 ' and '40' are row percentages per third party share; percentages in column 'Sum' are column percentages across choice options).

\begin{tabular}{ccccc}
\hline \multirow{2}{*}{ Choice Option } & \multicolumn{3}{c}{ Third Party Share } & \multirow{2}{*}{ Sum } \\
\cline { 2 - 4 } & $\mathbf{1}$ & $\mathbf{2 0}$ & $\mathbf{4 0}$ & \\
\hline Accept & $93(26.5 \%)$ & $130(37.0 \%)$ & $128(36.5 \%)$ & $351(46.1 \%)$ \\
Proposer-Reject & $149(39.0 \%)$ & $117(30.6 \%)$ & $116(30.4 \%)$ & $382(50.1 \%)$ \\
Third-Party-Reject & $12(41.4 \%)$ & $7(24.1 \%)$ & $10(34.5 \%)$ & $29(3.8 \%)$ \\
\hline
\end{tabular}

A multinomial logit model was applied to test differences in rejections depending on responder- and third party shares. The three options: acceptance, proposer-rejection and third party-rejection served as the levels of the dependent variable, with acceptance as the reference level. Responder share and third party share served as predictors, including an interaction term. The overall model fit was significant compared to the Null model according to a likelihood-ratio test, $\chi^{2}(12)=328, p<0.001$, McFadden- $\mathrm{R}^{2}=0.26$. In particular, rejections towards the proposer decreased significantly when the responder's share increased from 1 to 20 Euros $(b=-2.27, p<0.001)$. Furthermore, rejections towards the third party significantly decreased when the responder's share increased from 1 to 20 Euros ( $b=-2.19, p=0.001)$. However, an increase in third party share, be it from 1 to 20 or from 1 to 40 Euros, did not significantly change rejection behavior towards either player. Yet, the interaction between responder share and third party share turned out to be significant: if responder share and third party share both amounted to 20 Euros, proposer-directed rejections decreased significantly compared to responder and third party shares of 1 Euro $\left(b_{20,20}=-1.09, p=0.022\right)$, and they decreased even more if third party share amounted to 40 Euros $\left(b_{20,40}=-0.99, p=0.035\right)$.

In sum, rejections were targeted almost exclusively towards the proposer, and there was virtually no indication of envy-related rejections directed towards the third party. In fact, data suggest that the responders' propensity to punish the proposer diminishes if the responder receives 20 Euros and the third party also receives a share of 20 or 40 Euros. Third party rejections are not influenced by what the third party obtains. This partially confirms hypothesis one, as we assumed a default propensity to punish the proposer, but disconfirms the additional assumption that an increasing discrepancy of shares with respect to the third party will enhance the relative number of third party rejections.

Since third party rejection rate was particularly low, and was virtually unaffected by experimental manipulations, we collapsed third party rejections with proposer-rejections. The resulting binary accept/reject decision variable was analyzed as a function of the two independent variables: responder 
share and third party share, via a mixed-model logistic regression analysis [65], with the accept decision as the dependent variable and responder share (2-level factor: 1 vs. 20 Euros) and third party share (3-level factor: 1 vs. 20 vs. 40 Euros) as predictors, including an interaction term; participants were treated as a random factor.

The logistic regression analysis with acceptance as dependent variable yielded significant effects for responder share $(b=0.181, p<0.001)$ as well as a significant interaction term $(b=0.002, p=0.006)$. Acceptance rate was not related to third party share. As can be seen in Figure 1a, acceptance rate increased significantly when responder share increased from 1 to 20 Euros (average increase was from $16 \%$ to $76 \%$ acceptance). Note that if responder share was 20 , acceptance was highest when the third party's share was also 20 (85\% acceptance in contrast to $59.8 \%$ when the third party's share was only 1 Euro, this difference is significant as implied by the significant interaction term of the logistic regression). Acceptance rate dropped slightly but insignificantly when the third party share rose to 40 Euros. In contrast, acceptance rate was uniformly low when the responder's share was only 1 Euro, irrespectively of the third party share, and, by implication, also independently of the proposer share.

Figure 1. Study 1: (a) Acceptance rate; (b) Fairness; (c) Satisfaction as a function of shares; (d) Satisfaction as a function of response. Error bars indicate 95\% CI.

(a)

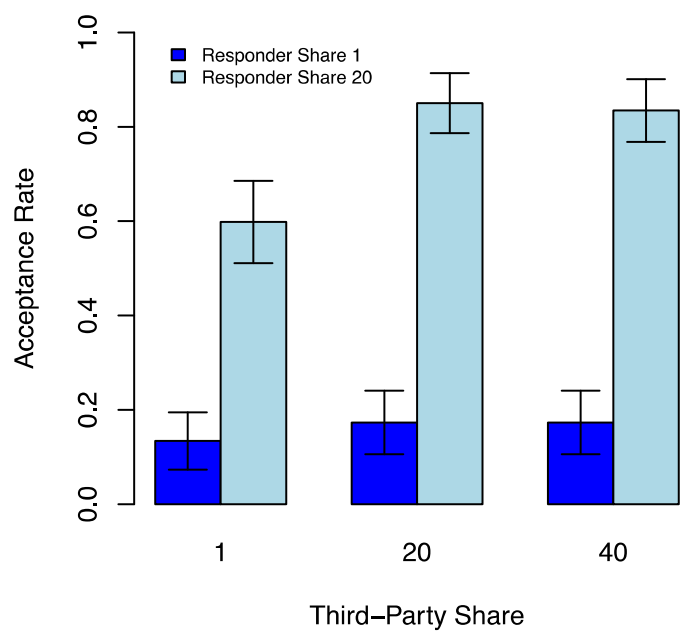

(c)

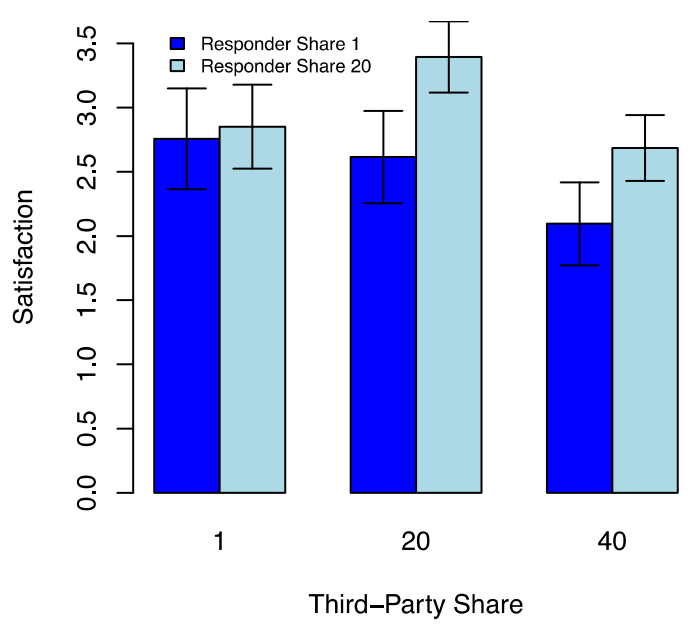

(b)

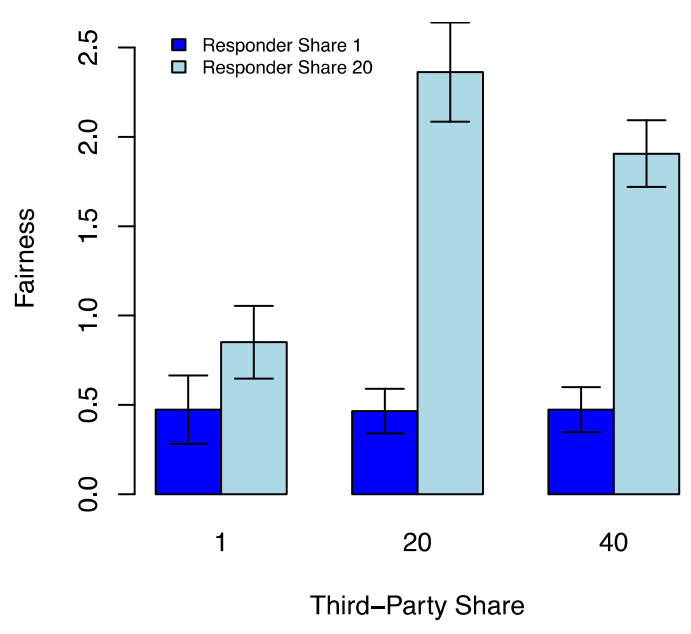

(d)

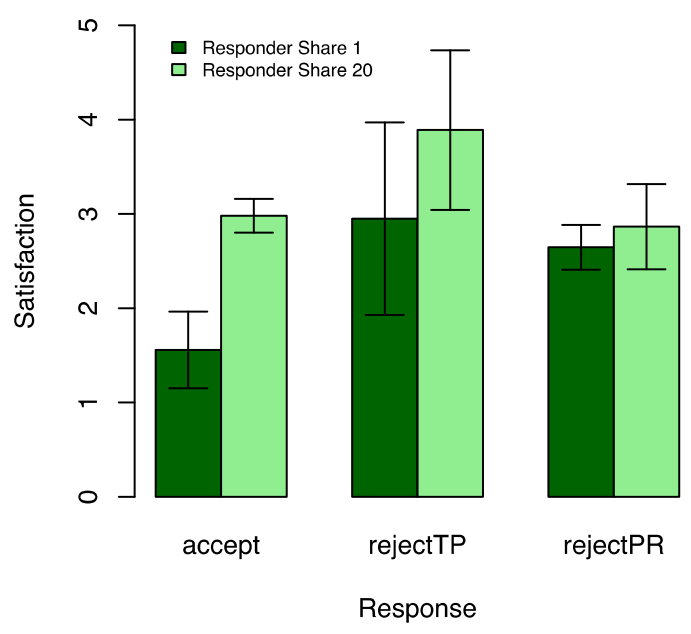


These findings are consistent with the multinomial logit analysis, and confirm that the likelihood of accepting a proposed share is primarily influenced by the absolute amount of the responder's own share, and further increases if this share is matched by an equal share for the third party.

In sum, we find that rejections are almost exclusively directed towards the proposer. This suggests that the responder's decision is primarily driven by inferences concerning the proposer's intention, and respondents reject in order to punish the proposer for proposing an unfair offer. Likelihood of acceptance increases when, in addition to the responder's perceived fair share, the third party is also offered a fair amount, suggesting that acceptance may be based on a judgment of overall distributional equality.

Perceived fairness. Responders were asked to directly rate how fair they considered the proposer's offer on a scale from zero to seven. A 2 (responder share) $\times 3$ (third party share) analysis of variance with repeated measurement on both independent variables shows a significant main effect of responder share, $F(1,126)=351.5, p<0.001, \eta_{\mathrm{G}}^{2}=0.248$ (we report generalized eta squared $\eta_{\mathrm{G}}^{2}$ as effect size statistic [66]), a significant main effect of third party share, $F(2,252)=47.9, p<.001, \eta_{\mathrm{G}}^{2}=0.079$, and a significant interaction, $F(2,252)=42.9, p<0.001, \eta^{2}{ }_{\mathrm{G}}=0.080$.

Figure $1 \mathrm{~b}$ shows that participants in the role of the active responder considered a share of 1 Euro as fundamentally unfair, irrespective of what was offered to the third party $\left(M_{\text {Fairness }}=0.47\right.$, on a 8-point rating scale). If responders were offered a share of 20 Euros, the increase in perceived fairness is substantial. Most importantly, fairness is maximal when the third party's share was also 20 Euros, and fairness decreased slightly but significantly if the third party's share further increased from 20 to 40 Euros $(M=1.91), t(125)=3.494, p<0.001$, now exceeding the responder's share by 20 Euros. This interaction pattern between responder and third party shares suggests that, in contrast to choice behavior, fairness judgments are affected by the relative discrepancy between responder share and third party share, and decrease significantly if the third party's share deviates in either direction from the responder's share, providing support for Hypothesis 1.

Perceived satisfaction. After having made their decision to accept or to reject, participants rated their satisfaction on a scale from zero to seven. Analogous to the previous analysis of fairness, we first tested whether satisfaction depends on responder and third party shares. A 2 (responder share) $\times 3$ (third party share) analysis of variance with repeated measurement on both independent variables shows a significant main effect of responder share, $F(1,126)=18.3, p<0.001, \eta_{\mathrm{G}}^{2}=0.018$, a significant main effect of third party share, $F(2,252)=11.7, p<0.001, \eta^{2}{ }_{\mathrm{G}}=0.019$, and a significant interaction, $F(2,252)=5.1, p<.007, \eta_{\mathrm{G}}^{2}=0.006$.

Figure 1c shows that satisfaction was influenced by a comparison process between the responder's share and the third party's share. Responder-to-third party share proportions of $1 / 1,20 / 1,1 / 20$, and 20/40 all led to moderate satisfaction ratings of about 2.7 (on a 8-point rating scale). Note that $1 / 1$ shares $(M=2.76)$ and $20 / 40$ shares $(M=2.69)$ are virtually identical concerning satisfaction ratings, whereas they differed markedly concerning acceptance decisions and fairness ratings. A pronounced positive peak in satisfaction is observed when both shares were high and equal, that is, with a 20/20 proportion $(M=3.39)$. If the third party received 40 Euros, the drop in satisfaction $(M=2.69)$ was significant, $t(125)=5.151, p<0.001$.

Secondly, as satisfaction was measured after the decision to accept or reject, we tested if satisfaction increases after rejections (Hypothesis 3). Due to the unbalanced design caused by different frequencies of accept and reject decisions, we applied a mixed-effects model [65] with participants as random 
effect, and response (accept, or reject towards proposer or third party) and responder share as independent variables. It turned out that in addition to a significant main effect of responder share $\left(\chi^{2}(1)=12.02, p<0.001\right)$, the main effect of response $\left(\chi^{2}(1)=12.39, p=0.002\right)$, as well as the interaction between responder share and response, is significant $\left(\left(\chi^{2}(2)=17.92, p<0.001\right)\right.$.

Figure 1d shows that satisfaction after rejecting is as high as after accepting an offer of 20 Euros; satisfaction drops substantially after accepting a low unfair offer of 1 Euro. This finding lends partial support to Hypothesis 3, confirming that a rejection, that is, a foregone gain of money, has the potential to regulate one's feelings of satisfaction up to a level which is comparable to an accepted gain of 20 Euros.

\subsection{Discussion}

In sum, three findings of Study 1 are noteworthy. First, and most important, rejections were almost exclusively targeted at the proposer. When responders chose to reject, that is, to voluntarily forego a monetary gain, in most cases they punished the proposer by selecting Type 1 rejections. The fact that a third party participant received a higher share was for the most part irrelevant for the responder's decision to reject. Acceptance rate even increased when third party shares increased, in particular when responder and third party shares were distributed evenly, suggesting a preference for equity as a driving motive; note that large and even shares for responder and third party also imply a reduced and similar share for the proposer.

Second, the decision to accept is largely based on the amount of money the responder receives, irrespective of the distribution of shares (Figure 1a). Low offers (1 Euro) lead to rejection, high offers (20 Euros) lead to acceptance. However, feelings of fairness and satisfaction deviate from this pattern. Perceived fairness as well as satisfaction depend considerably on the distribution of shares. Fairness is judged as high if both recipients, responder and third party, receive a comparable high share, but judged as low if either one receives a low share (Figure 1b). Feelings of satisfaction parallel this pattern, but are additionally influenced by third party comparisons, dropping as the third party's share increases (Figure 1c).

One could assume that an equal share between the three parties plays a prominent role as being especially fair, and downward deviations from the equal share lead to dissatisfaction and rejection. With a total amount of 100 Euros, an equal share would have been 33 Euros per player, and the 20 Euro share would have been unfair. Concerning rejections decisions, results suggest that this is not the case; however, fairness ratings reach only a maximum of 2.5 points on a 8 -point scale when responders obtain 20 Euros, indicating that a share of 20 is accepted, but not considered as fair.

Third, findings suggest that, as hypothesized, rejection plays a regulating role. Feelings of satisfaction after a rejection are as high as after accepting a large share, thus compensating for the actual monetary loss. Interestingly, satisfaction after accepting a low offer is relatively low (Figure 1d), suggesting that the low gain does not compensate for the missed opportunity to retaliate towards a malevolent proposer.

Study 1 has some shortcomings which we address in Study 2. First, as outlined above, the ultimatum game scenarios were purely hypothetical, and somewhat contrived; that is, no real monetary payoffs were paid contingent on participants' choices, and the introduction of factitious characters (Mister 
Weber and Paulsen) might not be able to elicit the relevant feelings. Since it is often claimed that real payments yield more valid results [62], in Study 2 participants were actually paid according to their choices. Second, only fairness and general satisfaction ratings were assessed in Study 1, but not the specific emotions anger and envy, which are focused in Study 2.

\section{Study Two}

Study 2 aimed to replicate the findings from Study 1, and to extend Study 1 in two important aspects. First, participants received real monetary payments depending on their actual choice in the ultimatum game. Second, we assessed ratings of specific emotions such as anger towards the proposer and envy towards the third party, in addition to satisfaction and perceived fairness. Also, in contrast to Study 1, Study 2 employs a complete between-subjects design, that is, each participant made only one choice concerning one particular ultimatum game scenario.

\subsection{Participants}

A total of 118 participants were randomly assigned to one of the six conditions of the 2 (responder share) $\times 3$ (third party share) between-subjects factorial design. Students from undergraduate psychology courses served as participants. They received credits as part of their course requirements, as well as monetary payoffs contingent on their choice behavior.

\subsection{Design}

The basic rules of the three-player, three-option ultimatum game employed in Study 2 were largely identical to Study 1. An anonymous proposer made an offer on how to distribute a total endowment of 20 Euros among him- or herself and two other players. Different to Study 1, the alleged proposer could not choose an arbitrary distribution among the three parties, but could only choose from a restricted set of distributions (Table 3). It has been shown that the available option set can have a significant impact on responders' attributions of intentions [15], but the available options in Study 2 were designed to represent the whole range from altruistic to malicious intentions, allowing for the expression of a variety of motives. As in Study 1, all participants were assigned to the role of the active responder; a second anonymous participant was declared as the passive third party. Unknown to the active participant, the offers were not made by real proposers, but manipulated by the experimenter.

Since participants were made to believe that the offers were made by a real person, although anonymous, this type of design involves deception. There is a long and ongoing controversy about possible detrimental effects of deception in experimental studies [62,67,68], and deception has been practically prohibited in genuine experimental economics [69]. However, empirical evidence showing that deception threatens the validity of experiments, for example, due to suspicion or second-thoughts of participants, is mixed at best [70]. With respect to the two prominent arguments, that (i) resentment of participants, and (ii) suspicion of being deceived, threatens validity and contaminates the participant pool, Hertwig and Ortmann [70] conclude that "..it seems fair to conclude that the issue of how pervasively deception raises resentment is not yet decided." (p. 69), and concerning the contamination of subject pools: "It seems fair to say that this did not happen." (p. 82). 
In particular, if a kind of ethically acceptable deception allows stronger experimental control, we consider it acceptable [68,71]. As we are primarily interested in the effects of specific distributions of shares, even if some are not very realistic, a systematic manipulation is only possible by means of deceiving participants about the true origin of an offer (or by means of hypothetical scenarios, as in Study 1). Following the classification reported in Hertwig and Ortmann [70], based on Sieber, Iannuzzo, and Rodriguez [72], our deception would qualify as 'bogus device' and 'role deception', that is, false information about stimulus material and false information about an interacting participant. The reason why we chose this approach is to exert maximum control about the independent variables, here the specific combination of responder share and third party share.

Two independent variables were varied in a 2 (responder share: low vs. medium) $\times 3$ (third party share: low vs. medium vs. high) between-subjects factorial design. The independent variables were manipulated by assigning one of six possible share distributions to each participant. The six distributions are shown in Table 3. Column 1 shows the amount the proposer keeps for him- or herself, Column 2 shows the amount allocated to the active responder and Column 3 shows the amount allocated to the third party. The share allocated to the active responder (that is, to the participant) was either low (1 Euro) or medium (5 Euros). The share allocated to the third party was either low (1 Euro), or medium (5 Euros), or high (9 Euros), yielding six experimental conditions. Note that as in Study 1 there is a dependency due to the fixed total amount of 20 Euros. The numerical shares were selected so that the correlation across conditions between responder share and third party share is zero, however, as a consequence proposer shares then correlate negatively with third party shares (rank correlation $=-0.80$ ) and with responder shares (rank correlation $=-0.46$ ). All participants were actually paid the payoff as presented, depending on their choices.

As a consequence of these pre-defined distributions of shares, some distributions might not be very realistic in a natural setting; in particular, distributions 3, 4 and 5 might appear as strange, because either the proposer kept less for himself than he allocated to the responder or the third party, or because more was allocated to a passive third party than to the active responder. Hence, we will test the essential hypotheses additionally with only the subset of participants who were assigned the more realistic share distributions 1,2 and 6 .

\subsection{Material and Procedure}

Participants received a two-page questionnaire. Page 1 presented general instructions about the rules of the ultimatum game and assigned the role of the active responder to the participant. Page 2 presented the response scales.

Participants were informed that the study consisted of two stages, and that they were now at Stage 2. Allegedly, at Stage 1, another person had been assigned the role of the proposer, and had made a real choice about how to share a total amount of 20 Euros between him- or herself and two anonymous responders. It was explained that in order to maintain anonymity, it was necessary to separate the two stages of the study.

The six available options were shown in tabular form, and one of the options was circled, ticked off or otherwise marked in handwriting with a blue pencil as if a real person had made a choice. There were no comments from participants that this scenario was perceived as contrived or not credible. The 
alleged choice constituted the experimental manipulation, that is, a particular distribution of shares (see Table 3).

Table 3. Distributions of offers available to the proposer to share an endowment of 20 Euros (textual labels as used in the text).

\begin{tabular}{cccc}
\hline Distribution & Proposer Share & Responder Share & Third Party Share \\
\hline 1 & 10 & 5 (medium) & 5 (medium) \\
2 & 18 & 1 (low) & 1 (low) \\
3 & 6 & 5 (medium) & 9 (high) \\
4 & 10 & 1 (low) & 9 (high) \\
5 & 14 & 1 (low) & 5 (medium) \\
6 & 14 & 5 (medium) & 1 (low) \\
\hline
\end{tabular}

After being prompted to turn to page 2, participants first copied the proposed distribution of shares that was marked on page 1; this was in order to make sure that they had read the correct option, and that the relevant values were fully in sight when they made their judgments and their own choice.

Then, participants indicated their ratings on 7-point rating scales: Fairness of the offer $(0=$ very unfair, $6=$ very fair $)$, envy toward the third party $(0=$ not at all, $6=$ very envious $)$, anger towards the proposer $(0=$ not at all, $6=$ very angry $)$, and general feeling at this moment $(0=$ very bad, $6=$ very good).

Note that the rating of envy is directed explicitly towards the third party, and the rating of anger towards the proposer. It could well be that participants also feel envy towards the proposer, and anger towards the third party, respectively. We are, however, interested in the specific emotion elicited by either the proposer or the third party; accordingly, the rating scales included the particular target person.

Following these ratings, participants decided how to respond to the proposed offer, that is, either to accept the offer, to reject towards the proposer, or to reject towards the third party. The resulting payoffs of each choice were listed beneath each option. After having made their choice, participants rated their general feeling a second time.

After completion, participants handed the questionnaire to the experimenter and received their respective payoffs (5 or 1 Euros upon acceptance, nothing upon rejection). Participants were informed that depending on their choice, the proposer as well as the third party would be paid their respective amounts of money. There was no indication whatsoever that participants detected the hypothetical character of the proposer and the third party.

\subsection{Results}

Acceptance and rejection. Overall, 79 out of 118 participants accepted the proposed offer (67\%), and 39 rejected (33\%). There was not a single rejection towards the third party (Type 2 rejection); that is, all rejections were targeted towards the proposer. As a consequence, all further analyses are based on a dichotomous choice variable (accept or reject).

As can be seen from Figure 2a, acceptance rate (computed as the proportion of acceptances per condition) increased with increasing responder share as well as with increasing third party share. A logistic regression analysis with acceptance as dependent binary variable, and responder and third 
party shares as independent variables, yielded significant effects for responder share $\left(\chi^{2}(1)=38.8\right.$, $p<0.001)$, and third party share $\left(\chi^{2}(2)=20.9, p<0.001\right)$; the interaction just missed significance $\left(\chi^{2}(2)=5.9, p=0.052\right)$. This pattern closely parallels the pattern in Study 1, except that acceptance rate also increased across third party shares when the responder received only the minimum amount of 1 Euro.

Figure 2. Study 2: (a) Acceptance rate; (b) Fairness; (c) Anger; (d) Envy. Error bars indicate $95 \% \mathrm{CI}$.

(a)

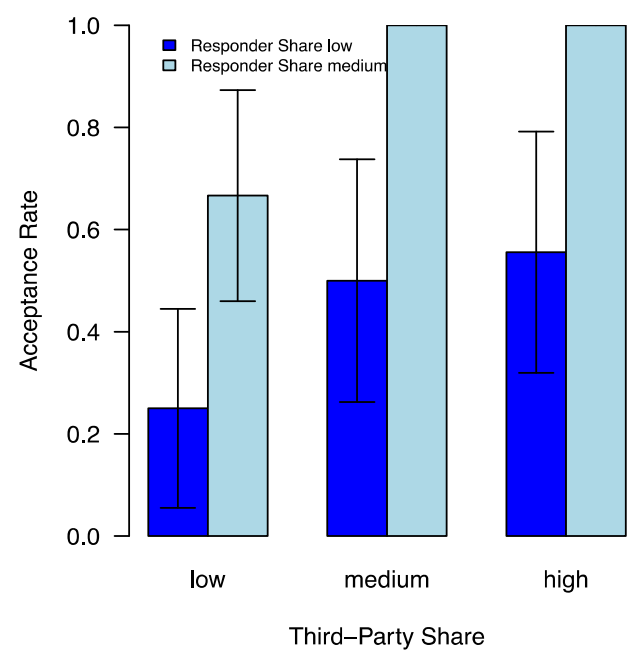

(c)

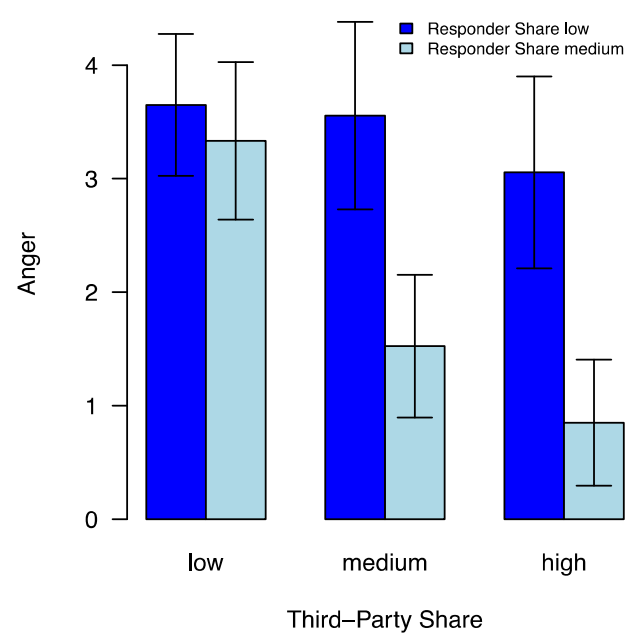

(b)

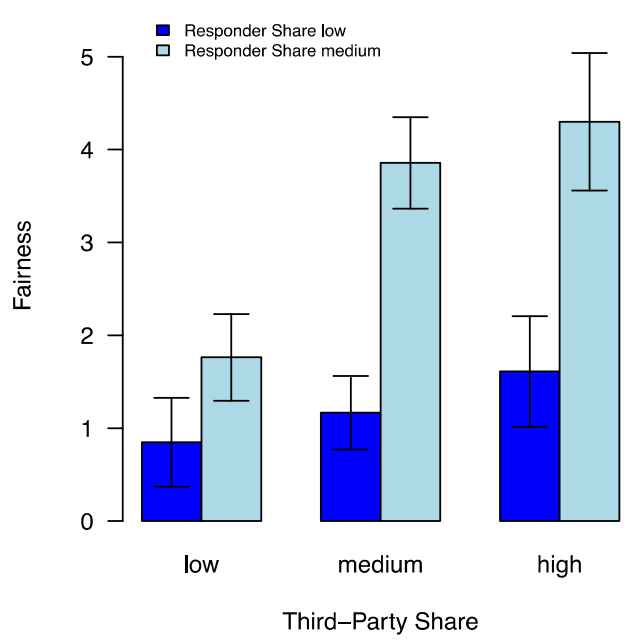

(d)

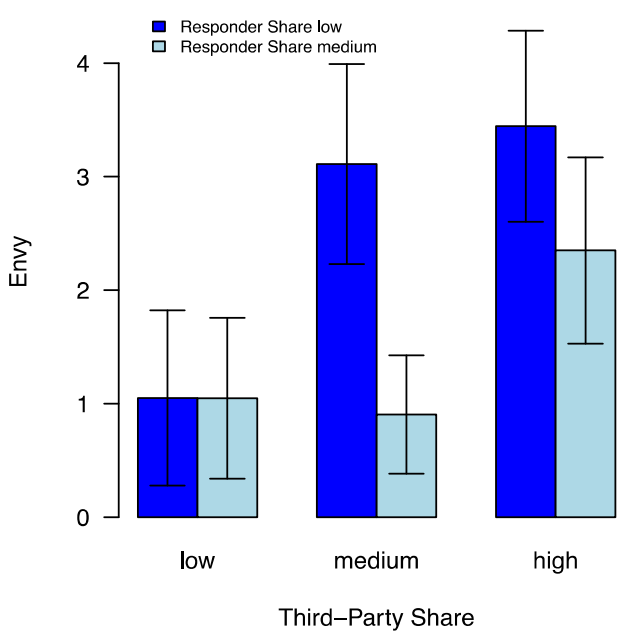

As mentioned above, it can be argued that from the six available distributions (Table 3) some are unlikely to occur in reality. In distribution 3 the proposer gives a larger share to the third party than to himself, and in distributions 4 and 5 the third party obtains a larger share than the active responder. These somewhat strange allocations might surprise the participants, lead to confusion or atypical emotional reactions and distort the results. Thus, we focused on the more realistic distributions 1, 2 and 6. In particular, we compared distributions 1 and 6 to test the effect of a change in third party share, and we compared distributions 2 and 6 to test the effect of a change in responder share; in both 
comparisons, the third party never obtains more than the responder, and the proposer always keeps a substantially larger share.

We compared the proportion of acceptances under distributions 6 and 1, testing the hypothesis that acceptances are less likely under distribution 6 if the third party share drops below the responder's share, creating an unfair offer. A test for equality of proportions yields a significant difference, $\chi^{2}(1)=6.17, p=0.013$, indicating that acceptance rate decreases if third party share drops relative to responder share. Second, we compared acceptances under distribution 6 and 2, testing the hypothesis that acceptances are less likely under distribution 2 if responder share is low, even if third party share is equally low. A test for equality of proportions yields a significant difference, $\chi^{2}(1)=5.57, p=0.018$, indicating that acceptance rate decreases if responder share is very low, even if equality with the third party is maintained. These results corroborate the conclusion that responders accept the proposal if their share is not low, and if the third party share is equal, given the additional realistic fact that the proposer's share is always somewhat larger.

Perceived fairness. A $2 \times 3$ analysis of variance with fairness rating as the dependent variable and responder share (low versus medium) and third party share (low versus medium versus high) as independent variables yielded a significant main effect for responder share, $F(1,112)=86.18$, $p<0.001, \eta_{\mathrm{p}}^{2}=0.44$ (partial eta-squared), a significant main effect for third party share, $F(2,112)=19.35, p<0.001, \eta_{\mathrm{p}}^{2}=0.26$, as well as a significant interaction, $F(2,112)=7.04, p=0.001$, $\eta_{p}^{2}=0.11$ (Levene's test of homogeneity of variance is $n s, p=0.713$ ). Figure $2 \mathrm{~b}$ shows that perceived fairness was consistently greater when the responder obtained a medium compared to a low share. Furthermore, fairness increased with increasing third party shares. The interaction indicates that fairness was particularly large if the third party also obtained a medium or a high share. In contrast to Study 1, there was no drop in fairness when the third party received more than the responder.

Following the same logic as we did with respect to acceptance rate, we compared distributions 6, 2 and 1 because these distributions are likely to occur under realistic circumstances. In particular, we tested if fairness is lower in distribution 2 compared to distribution 6. A Tukey HSD test indicates that the difference is not significant, $\Delta=0.911, p=0.171$. Next, we tested if fairness is lower in distribution 6 compared to distribution 1; a Tukey HSD test yields a significant difference with $\Delta=-2.095$, $p<0.001$. These results qualify the above interaction effect, suggesting that also under realistic proposals, it is perceived as unfair if the third party obtains a low share, irrespective of responder share, and that it is perceived as especially fair if both responder and third party obtain the same medium share.

Anger towards the proposer. A $2 \times 3$ analysis of variance with the rating of anger towards the proposer as the dependent variable and responder share (low versus medium) and third party share (low versus medium versus high) as independent variables yielded a significant main effect for responder share, $F(1,112)=27.43, p<0.001, \eta_{\mathrm{p}}^{2}=0.20$, a significant main effect for third party share, $F(2,112)=9.68, p<0.001, \eta^{2}=0.15$, as well as a significant interaction, $F(2,112)=4.42, p=0.014$, $\eta^{2}=0.07$ (Levene's test of homogeneity of variance is $n s, p=0.728$ ). Figure $2 \mathrm{c}$ shows that if responders obtained a medium share, anger towards the proposer dropped if third party shares increased. If a low share of only 1 Euro was allocated to the responder, however, anger persisted on a high level, irrespective of third party shares. 
We test again differences in anger for the realistic distributions 6, 2 and 1. Comparing distributions 2 and 6 we expect that anger towards the proposer is higher in distribution 2 due to the low share of 1 Euro only. However, a Tukey HSD test yields no significant difference $(p=0.987)$, indicating that anger persists even if the responder obtains a medium share, as long as the third party does not receive a medium share. Comparing distribution 6 and 1, we assume a substantial drop in anger if the third party receives a medium share in contrast to a low share, given that the responder obtains a medium share. A Tukey HSD test yields a significant difference, $\Delta=-1.809, p=0.004$, indicating that anger decreases substantially if both responder and third party obtain an equal medium share.

Envy towards the third party. A $2 \times 3$ analysis of variance with the rating of experienced envy towards the third party as the dependent variable and responder share (low versus medium) and third party share (low versus medium versus high) as independent variables yielded a significant main effect for responder share, $F(1,112)=12.14, p=.001, \eta_{p}^{2}=0.10$, a significant main effect for third party share, $F(2,112)=11.49, p<0.001, \eta_{\mathrm{p}}^{2}=0.17$, and a significant interaction, $F(2,112)=4.13, p=0.019$, $\eta_{\mathrm{p}}^{2}=0.07$ (Levene's test for homogeneity of variance is $n s, p=.551$ ). As can be seen in Figure $2 \mathrm{~d}$, envy increased substantially whenever the third party share exceeded the responder's share; envy was highest in the condition with the largest difference, that is, when the responder share was low and the third party share was high.

Figure $2 \mathrm{~d}$ shows that envy is only high in distributions 3,4 , and 5; that is, under the three more realistic distributions 6,2, and 1 we would not expect envy to occur, because the third party never obtains more than the responder. Accordingly, under all three distributions envy is consistently low, and no significant difference between any pair of realistic distributions can be found.

Causes of anger and envy. We expected anger to be a function of the difference between responder share and the share that the proposer kept to him- or herself. In contrast, envy was expected to be a function of the difference between responder share and third party share. Two difference variables were computed: the difference between the responder's share minus the proposer's share, and the difference between the responder's share minus the third party share (the two differences are not substantially correlated due to the construction of the provided distributions, see Table 3). A linear regression analysis with anger as the dependent variable and proposer difference and third party difference as predictors yielded a significant negative regression coefficient only for proposer difference $\left(b=-0.19, p<0.001, R_{\text {adjusted }}^{2}=0.27\right)$. In contrast, a linear regression analysis with envy as the dependent variable yielded a significant negative regression coefficient only for third party difference $\left(b=-0.243, p<0.001, R_{\text {adjusted }}^{2}=0.21\right.$ ). This confirms our hypothesis that anger is triggered by a comparison with the proposer, whereas envy is triggered by a comparison with the third party.

Acceptance and rejection as a function of emotions. Having established that anger and envy are systematically related to relative discrepancies in shares, we analyzed if emotions can predict the acceptance decisions of responders. A logistic regression analysis with the binary accept/reject variable as criterion and fairness, anger and envy as predictors yielded a significant regression coefficient for fairness $\left(b=0.715, s e_{b}=0.215, p<0.001\right)$, and anger $\left(b=-0.396, s e_{b}=0.163, p=0.015\right)$. The fairer an offer was judged, the more likely it was to be accepted. The angrier responders felt towards the proposer, the less likely they were to accept the offer. Envy towards the third party, however, was not significantly related to rejection rate. 
As above, and as a kind of validity check, we computed the same model but including only observations from the more realistic distributions 6,2 , and 1 (Table 3). The logistic regression analysis yielded a significant coefficient for fairness $\left(b=0.751, s e_{b}=0.287, p=0.009\right)$. The coefficient for anger did not reach significance $\left(b=-0.385, s e_{b}=0.246, p=0.118\right)$. Although anger is not a significant predictor, due to the reduced sample size $(n=62)$, the size of the coefficients as well as the size of the standard errors are largely unaffected, indicating that the result is presumably not due to weird emotional reactions concerning unrealistic proposals.

As pointed out earlier, acceptance rate was directly influenced by the distribution of shares. Employing a mediation analysis [73], we tested whether anger mediates the relationship between offer and decision. We assumed that the perception of an unfair discrepancy in shares between responder and proposer (independent variable) would lead to anger (mediator), which in turn would increase the tendency to reject the offer (dependent variable). The mediation analysis showed that all relevant mediating conditions are fulfilled. In particular, the direct effect of share discrepancy on rejection decreased when anger was included as a mediator (from $c=0.05$ to $c^{\prime}=0.038$ ), and the indirect effect of share discrepancy on rejection via anger was significant according to a Sobel test (indirect effect $a b=0.0119, \mathrm{z}=2.595, p=0.005)$, confirming the mediating role of anger.

Feelings after rejection. The participants' general feeling was assessed twice on a bipolar scale ranging from very bad to very good, once before and once after they had made their choice. The change in feeling, as a function of choice, was tested with a 2 (Time: before versus after choice) $\times 2$ (Choice: acceptance versus rejection) analysis of variance with repeated measurement on Time (Figure 3). Results show a significant main effect of Time, $F(1,116)=35.2, p<0.001$, and a significant main effect of Choice, $F(1,116)=11.5, p<0.001$, indicating that participants generally feel better after having made a choice, and that before as well as after the choice they feel better when accepting the offer. The interaction between Time and Choice just fails to reach significance, $F(1,116)=3.3, p=0.071$.

Figure 3. Study 2: General feeling ( $0=$ very bad, $6=$ very good) before and after the choice to accept or reject. Error bars indicate $95 \%$ CI.

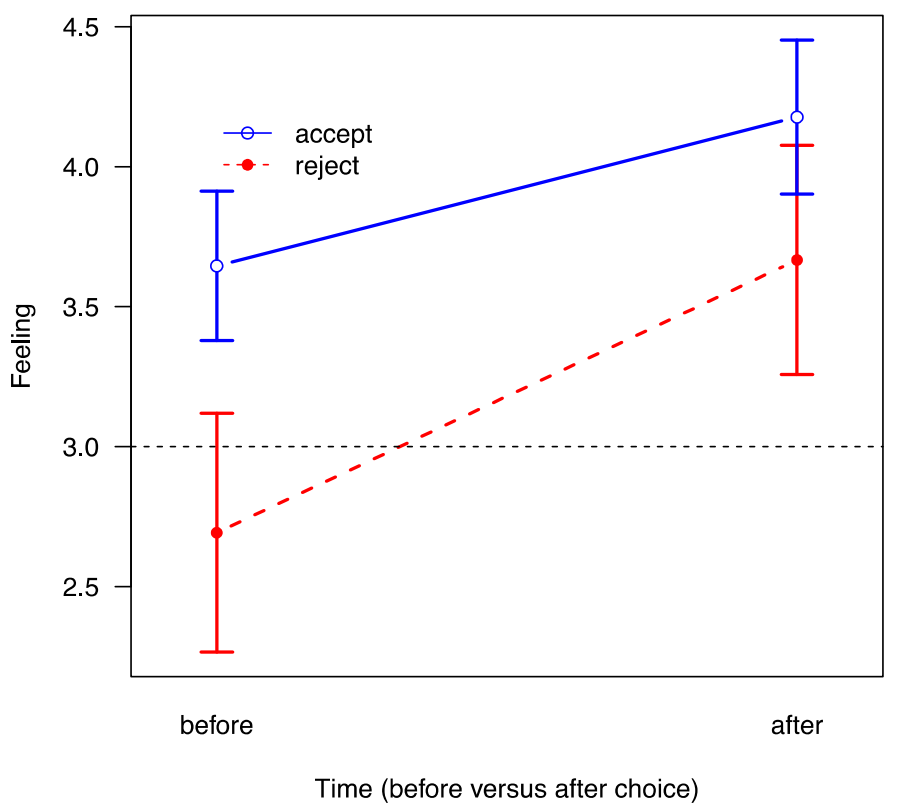


As above, we ran the same analysis of variance with the subset of participants from the three realistic conditions with pre-defined distributions 6, 2, and 1 (Table 3). The results are virtually unchanged, yielding a significant effect of Time, $F(1,60)=20.9, p<0.001$, a significant effect of Choice, $F(1,60)=9.8, p=0.003$, and no interaction effect.

A closer look at Figure 3 suggests that responders feel substantially better even after having made a reject decision, thereby moving from the neutral zone (the midpoint of the bipolar feeling scale is 3.0) into the positive zone. Those responders who will reject do not feel significantly different from the neutral point before making the choice $(M=2.69, t(38)=-1.41, p=0.166)$, whereas after having actually rejected, their general feeling has turned significantly positive $(M=3.67, t(38)=3.19$, $p=0.003$ ). In contrast to Study 1 , however, acceptance is always associated with a more positive overall feeling state.

\subsection{Discussion}

The results of Study 2 for the most part replicated the findings of Study 1. Most important, rejections were exclusively targeted at the proposer, and never towards the third party. Also, perceived fairness increased with rises in responder share as well as in third party share, indicating that fairness is judged in a holistic way and not merely focused on the responder. An equal and especially fair share would have been an offer of 6 or 7 Euros for each party; the actual maximum responder share of 5 Euros, however, seems to be sufficient to induce acceptance, and also to increase fairness ratings substantially. Yet, as in Study 1, there is leeway for fairness since fairness ratings barely exceed a mean of four points on an 8-point rating scale.

Nonetheless, responders felt considerable envy when third party share exceeded their own share, whereas anger was specifically associated with discrepancies between responder and proposer shares. Also, when comparing feelings before and after a choice was made, acceptance and rejection lead to more positive feelings after the choice, with rejections especially moving responders' feelings from neutral to positive.

Note that in contrast to Study 1, in Study 2 the set of available options for the alleged proposer was restricted to six distributions (Table 3). Although these distributions represent a mixed set, including 'fair' as well as 'malicious' distributions, we cannot rule out that changing the proposer options to a restricted set may change the participants' inferences about the proposer's intentions [15] (see also [74]). As some of these distributions can be considered unrealistic, in particular distributions 3, 4 and 5, we conducted specific analyses using only the subset of realistic distributions 1,2 and 6; however, results turned out to be virtually unchanged.

Study 2 extended the findings of Study 1 in several ways. We generally found that the provision of real monetary payoffs, compared to hypothetical scenarios, did not substantially change responder behavior, though acceptance rate increased slightly. The measurement of two specific emotions, anger and envy, yielded support for the hypothesis that emotions play an important role in ultimatum game behavior, with anger being especially important as a mediator for rejections.

Anger resulted from offers which allocated a higher share to the proposer than to the responder. As a consequence, anger mediated the decision to reject and, thus, to forego a monetary gain. Envy, on the other hand, resulted from offers which allocated a relatively higher share to the third party than to the 
responder. Envy, though, did not lead to rejections. This parallels the dissociation of satisfaction from choice found in Study 1: Responders admitted bad feelings when recognizing an unfair share compared to what the third party obtained, but this did not translate into penalizing behavior towards the third party. Evidently, rejections are mainly determined by reciprocity concerns towards the proposer; the proposer is the one who is held responsible and therefore punished, even at a cost to oneself.

\section{General Discussion}

The ultimatum game, together with other games such as the prisoners' dilemma, has yielded ample evidence that human behavior is often fundamentally at odds with predictions from classical game theory [75]. Presumably, strategic interactions trigger a broad array of emotional reactions which influence actual behavior in addition to and beyond the pure maximization of individual monetary payoffs [13]. The overall behavioral dynamics are commonly interpreted in terms of fairness, reciprocal altruism and violations thereof $[15,34,36]$. However, the specific psychological mechanisms which drive the relevant behaviors are only beginning to be understood.

Rejections in the ultimatum game are a case in point. Why do responders accept a monetary loss, even if it is a one-shot game, and the other player(s) are never actually encountered? One cause could be that people simply follow a social or moral norm, which recommends behaving in a certain way $[17,76]$. We assume that for social norms, and in particular for moral norms, the underlying psychological mechanism is an emotional one, as is suggested by an increasing amount of findings from behavioral game theory [12,25], behavioral decision research [8,9], and neuroeconomics [13,77]. We assume that responders in the ultimatum game experience specific emotions which are triggered by the received offer and an ensuing construal about presumed causes, and that the emerging emotions constitute the proximate causes of behavior. A low share and a large discrepancy in favor of the proposer elicit anger, and anger, in turn, motivates the responder to punish the proposer. A large discrepancy in favor of another third party elicits envy, which then may or may not motivate a decision to penalize the third party, depending on the circumstances. Punishing the proposer or a third party may then have consequences for the responder, for example, make him or her feel better after having experienced an unfair offer.

In order to test these assumptions, a new version of a three-player, three-option ultimatum game was devised. In this version, the proposer divides the endowment among three players: him- or herself, the active responder, and a passive third party. The responder may then react in one of three ways: accept the offer, reject towards the proposer or reject towards the third party. A proposer-directed rejection will yield nothing for the proposer and the responder, but the third party will keep her or his share. A third-party-directed rejection will yield nothing for the third party and the responder, but the proposer will keep his or her share.

It turned out that the responders virtually never punished the third party. That is, if responders rejected, they rejected towards the proposer. We further found that acceptance rate was especially low when the responder's absolute share was very low. Acceptance rate increased with increasing absolute payoffs for the responder, but also partially with an increasing share for the third party, indicating a concern for equity (Figures 1a and 2a). For some decades [78,79] it has been argued that a concern for 
fairness might be stronger in some circumstances than pure self-interest. In accordance with this claim, our results show that acceptance rate and judgments of fairness are indeed related.

Results further indicate that responders reject an offer when they are angry. Anger arises when the proposer keeps a disproportionately large share for him- or herself. We found that anger is a key mediating variable between the proposed distribution of shares and the responder's decision to reject. This suggests that the enforcement of fairness norms may be contingent on the elicitation of a relevant emotion, such as anger. Players who are not sufficiently angry might endorse the social norm of fairness, but not act on it. This conclusion is in line with the findings of Srivastava et al. [14], who were the first to clearly demonstrate the crucial role of anger as one cause of rejections, if targeted at the proposer.

Both our studies show that responders feel envy and dissatisfaction when the third-party share exceeds their own share. Interestingly, these feelings seem to have no substantial impact on their decisions to reject. Specifically, responders do not feel inclined to deprive the third party of their larger share, although this seems to be the source of their envy. This contrasts with the finding that proper envy is commonly accompanied by a tendency to harm the envied person, and to eliminate his or her envied possessions or attributes [55]. The tendency to punish the third party may not have shown up in our study, because although the third party's larger share elicits envy, the third party is not held directly responsible for the distribution of the shares.

However, the proposer, who is responsible for the distribution, is also not punished on the basis of envy; envy did not predict rejections towards the proposer. Third-party shares are effectual in causing envy, but these feelings do not seem to drive the decision to accept or to reject. What seems essential for the decision is the particular feeling of anger towards the proposer, who is probably held accountable for having brought about one's displeasure with the situation.

We found some indication that rejections serve to regulate responders' affective state. In Study 1, rejections towards the third party as well as towards the proposer yield a level of satisfaction comparable to the satisfaction attained from accepting a fair offer of 20 Euros. In Study 2, rejections even make responders feel good after the rejection when they felt neutral before. A number of emotion regulation mechanisms are known [59], and rejection might be an appropriate mechanism in the ultimatum game. One may even speculate that anger or envy are emotions people actually prefer to positive emotions when treated unfairly, because they help to act in a way which promotes their goals in the long run [80].

A number of shortcomings of our studies need to be pointed out. To begin with, some of the demands of experimentation in orthodox experimental economics have not been met: Do not use hypothetical choices, provide actual payoffs, and do not deceive participants $[69,81]$. In Study 1, the choice task was hypothetical, and no real payoffs were paid. We think it is an empirical question if hypothetical rewards threaten validity, and Bardsley et al. [82] conclude that there is no general answer to this question. A principled prohibition of hypothetical scenarios, however, does not seem justified. A recent study by Locey, Jones, and Rachlin [63] found no evidence that hypothetical rewards make a difference in social experiments, and conclude "...that results of experiments with hypothetical rewards validly apply in everyday life."

In Study 2, participants were deceived with respect to the alleged existence of a proposer. We consider these principles as desirable, but not as mandatory for experimental research. Evidently, many 
key findings in social psychology necessarily relied on deception [83], and seminal works in behavioral economics relied on hypothetical scenarios without actual payments [84]; Hertwig and Ortmann [62,67,70] discuss these issues (see also [82] for an in-depth discussion of deception). Following the classification reported in [70] (based on [72]), our kind of deception in Study 2 would qualify as 'bogus device', that is, false information about stimulus material and 'role deception', that is, false information about an interacting participant. As outlined above when describing Study 2, we consider it acceptable to make a trade-off between non-deception and experimental control in favor of experimental control in this study.

Hertwig and Ortmann [70] present a very balanced review of the issue, emphasizing that they themselves advocate not to use deception. However, from the available empirical evidence, they concede that it is at best an open issue if deception actually causes resentment, or contaminates potential subjects, and as a consequence, distorts experimental findings.

A more serious weakness of our version of a three-person ultimatum game is the dependency between proposer share, responder share, and third party share. Across conditions, increasing responder or third party shares are necessarily correlated with decreasing proposer shares, since the total amount is fixed. Even though our results showed the predicted pattern in that anger was related to responder shares and envy to third party shares, the interpretation is not unambiguous, since it is actually the unique configuration of the entire payoff distribution that determines participants' responses. But then, it is a defining characteristic of the ultimatum game that it is a quasi-zero-sum game; any gain for one party is a loss for some other party. Keeping, for example, proposer payoff constant, while varying third party share, would constitute another type of game with a variable endowment to share. This ambiguity would also apply to the traditional two-player ultimatum game: A responder who rejects, say, an offer of 20 (from a total of 100), might reject because he obtained too little, or because the proposer obtained too much (80 from 100).

Using pre-defined allocations of shares between proposer, responder and third party yields distributions of allocations which are not to be expected under realistic circumstances when people are free to specify any distribution. For example, in Study 2, Option 3 was a distribution of shares assigning less money to the proposer (6 Euros) than to the third party (9 Euros). Participants might be surprised, judge the proposer to be somewhat silly, and respond in unintended ways. We tried to counteract this objection by analyzing only the subset of realistic conditions, obtaining largely the same results as with the entire sample including the unrealistic conditions.

We assessed emotions via simple rating scales. Although this kind of direct measurement via self-report has turned out to be generally valid [85], there is certainly room for improvement. However, any kind of multimodal measurement, including physiological parameters or facial expression assessments, entails other difficulties, since it is currently unclear whether emotions yield coherent patterns across indicators of different modality [86]. Thus, for the sake of simplicity, we confined emotion assessment to simple self-reports, but agree that more sophisticated measurement instruments may be desirable in future research.

Another aspect, which we did not examine, is the possibility of interindividual differences in dispositions to respond in typical ways. For example, participants might differ in social value orientation [87]; with a competitive disposition they might be especially prone to envy, whereas participants with a more pro-social disposition might not be susceptible to envy or even to anger. 
More generally, a serious limitation of this study is the fact that we used a typical student sample, and this of course is a threat to external validity and generalization. Henrich, Heine, and Norenzayan [88] convincingly argue that participants they label WEIRD (western, educated, industrialized, rich, democratic) are highly atypical of the human species. Our samples certainly are weird in that sense; as a consequence, we can only generalize to this particular population.

In sum, by using the extended three-player, three-option ultimatum game, we were able to shed some light on the issue of what motivates responders to reject an offer. Our results suggest that it is mainly the motivation to punish the proposer, mediated by anger about an unfair offer. It seems less likely that responders care about equity per se when deciding to reject. This is not to say that responders are immune to feelings of envy, or generally, to displeasure arising from unfavorable comparisons with a third party. Quite to the contrary, we found that some comparisons trigger considerable envy, but these feelings did not predict behavior. More generally, when a situation elicits several specific emotions, maybe only one of them becomes relevant in guiding one's choices; other emotions, albeit experienced, may not be translated into behavior [9].

Envy might be a special case of a taboo emotion, at least in western cultures. Smith and Kim [55] emphasize that envy is an emotion that many people would not admit in public, and would not even acknowledge in private, let alone act on it. Anger, on the other hand, is an emotion commonly considered as socially acceptable, if it can be sufficiently justified and controlled. In the ultimatum game, a greedy proposer may well serve as a legitimate target for anger, but may also serve as a target for envy in order to enable an emotion regulation process.

Emotions such as anger or envy are triggered by a variety of contextual features and are subjectively construed by processes of recollection and interpretation [40]. It seems unlikely that the determinants of choice behavior in ultimatum game situations can be sufficiently modeled by a single utility function which relies solely on the payoff distribution $[34,89]$. We rather assume that specific emotions function as the proximate causes of behavior, and that the appraisals leading to emotional experiences build on information that is not entirely captured by utilities from payoffs. Furthermore, our data suggest that more than one emotion may be experienced at a time, causing conflicting tendencies to behave. Conflicting emotions and ambivalence, however, are not easily captured by a uniform utility function [9].

Our findings provide further evidence for the important role that specific emotions play in strategic interactions. Emotions such as anger may override pure monetary interests, and may thus help to establish fairness norms. We would speculate that, depending on the context, other emotions might play a similar role. For example, if a third party acts in an unethical way to receive a higher share, people might feel indignation and punish the third party rather than the proposer. Also, little is known about the time course of emotional activation; for example, responders might possibly regret their rejection as soon as their anger has cooled off $[90,91]$. Studying the dynamics of specific emotions across time thus seems to be a promising avenue to understand people's behavior in strategic decision situations.

\section{Acknowledgments}

We thank Johannes Daubenmerkl for collecting data in Study 1 and for providing many valuable suggestions. We thank three anonymous reviewers for their thorough reviews and many helpful recommendations. 


\section{Note}

The questionnaire (English translation) and the data are available at http:/www.ruedigerpfister.de/Games.

\section{References}

1. Camerer, C.F. Behavioral Game Theory. Experiments in Strategic Interaction; Russell Sage Foundation: New York, NY, USA, 2003.

2. Gächter, S. Behavioral game theory. In Blackwell Handbook of Judgment and Decision Making; Koehler, D.J., Harvey, N., Eds.; Blackwell: Oxford, UK, 2004; pp. 485-503.

3. Camerer, C.F. Behavioural studies of strategic thinking in games. Trends Cogn. Sci. 2003, 7, $225-231$.

4. Güth, W.; Tietz, R. Ultimatum bargaining behavior: A survey and comparison of experimental results. J. Econ. Psychol. 1990, 11, 417-449.

5. Güth, W.; Schmittberger, R.; Schwarz, B. An experimental analysis of ultimatum bargaining. J. Econ. Behav. Organ. 1982, 3, 367-388.

6. Akerlof, G.A.; Shiller, R. Animal Spirits: How Human Psychology Drives the Economy, and Why It Matters for Global Capitalism; Princeton University Press: Princeton, NJ, USA, 2009.

7. Bechara, A.; Damasio, A.R. The somatic marker hypothesis: A neural theory of economic decision. Games Econ. Behav. 2005, 52, 336-372.

8. Loewenstein, G.; Lerner, J.S. The role of affect in decision making. In Handbook of Affective Science; Davidson, R.J., Goldsmith, H.H., Scherer, K.R., Eds.; Oxford University Press: Oxford, UK, 2003; pp. 619-642.

9. Pfister, H.-R.; Böhm, G. The multiplicity of emotions: A framework of emotional functions in decision making. Judgm. Decis. Mak. 2008, 3, 5-17.

10. Blount, S. When social outcomes aren't fair-The effect of causal attributions on preferences. Organ. Behav. Hum. Decis. Process. 1995, 63, 131-144.

11. Haselhuhn, M.P.; Mellers, B.A. Emotions and cooperation in economic games. Cogn. Brain Res. 2005, 23, 24-33.

12. Pillutla, M.M.; Murnighan, J.K. Unfairness, anger, and spite: Emotional rejections of ultimatum offers. Organ. Behav. Hum. Decis. Process. 1996, 68, 208-224.

13. Sanfey, A.G.; Rilling, J.K.; Aronson, J.A.; Nystrom, L.E.; Cohen, J.D. The neural basis of economic decision-making in the ultimatum game. Science 2003, 300, 1755-1758.

14. Srivastava, J.; Espinoza, F.; Fedorikhin, A. Coupling and decoupling of unfairness and anger in ultimatum bargaining. J. Behav. Decis. Mak. 2009, 22, 475-489.

15. Falk, A.; Fehr, E.; Fischbacher, U. Testing theories of fairness-Intentions matter. Games Econ. Behav. 2008, 62, 287-303.

16. Gross, J.J. Handbook of Emotion Regulation; Guilford Press: New York, NY, USA, 2007.

17. Andreoni, J.; Bernheim, B.D. Social image and the 50-50 norm: A theoretical and experimental analysis of audience effects. Econometrica 2009, 77, 1607-1636. 
18. Güth, W.; Schmidt, C.; Sutter, M. Bargaining outside the lab-A newspaper experiment of a three-person ultimatum game. Econ. J. 2007, 117, 449-469.

19. Henrich, J.; Boyd, R.; Bowles, S.; Camerer, C.; Fehr, E.; Gintis, H.; McElreath, R.; Alvard, M.; Barr, A.; Ensminger, J.; et al. 'Economic man' in cross-cultural perspective: Behavioral experiments in 15 small-scale societies. Behav. Brain Sci. 2005, 28, 795-855.

20. Bosman, R.; van Winden, F. Emotional hazard in a power-to-take experiment. Econ. J. 2002, 112, 147-169.

21. Böhm, G.; Pfister, H.-R. Consequences, morality, and time in environmental risk evaluation. J. Risk Res. 2005, 8, 461-479.

22. Van Winden, F. Affect and fairness in economics. Soc. Justice Res. 2007, 20, 35-52.

23. Peters, E. The functions of affect in the construction of preferences. In The Construction of Preference; Lichtenstein, S., Slovic, P., Eds.; Cambridge University Press: New York, NY, USA, 2006; pp. 454-463.

24. Ortony, A.; Clore, G.L.; Collins, A. The Cognitive Structure of Emotions; Cambridge University Press: Cambridge, MA, USA, 1988.

25. Fehr, E.; Gächter, S. Altruistic punishment in humans. Nature 2002, 415, 137-140.

26. Kirchsteiger, G. The role of envy in ultimatum games. J. Econ. Behav. Organ. 1994, 25, 373-389.

27. Bosman, R.; Sutter, M.; van Winden, F. The impact of real effort and emotions in the power-to-take game. J. Econ. Psychol. 2005, 26, 407-429.

28. Pfister, H.-R.; Böhm, G. The function of concrete emotions in rational decision making. Acta Psychol. 1992, 80, 199-211.

29. Bolton, G.E.; Ockenfels, A. ERC: A theory of equity, reciprocity, and competition. Am. Econ. Rev. 2000, 90, 166-193.

30. Fehr, E.; Schmidt, K.M. A theory of fairness, competition, and cooperation. Q. J. Econ. 1999, 114, 817-868.

31. Falk, A.; Fischbacher, U. A theory of reciprocity. Games Econ. Behav. 2006, 54, 293-315.

32. Rabin, M. Incorporating fairness into game theory and economics. Am. Econ. Rev. 1993, 83, 1281-1302.

33. Bereby-Meyer, Y.; Niederle, M. Fairness in bargaining. J. Econ. Behav. Organ. 2005, 56, 173-186.

34. Bolton, G.E.; Ockenfels, A. A stress test of fairness measures in models of social utility. Econ. Theory 2005, 25, 957-982.

35. Dickinson, D.L. Ultimatum decision-making: A test of reciprocal kindness. Theory Decis. 2000, $48,151-177$.

36. Kagel, J.H.; Wolfe, K. Tests of fairness models based on equity considerations in a three-person ultimatum game. Exp. Econ. 2001, 4, 203-220.

37. Tyler, T.R. Social justice: Outcome and procedure. Int. J. Psychol. 2000, 35, 117-125.

38. Tyler, T.R.; Blader, S.L. The group engagement model: Procedural justice, social identity, and cooperative behavior. Pers. Soc. Psychol. Rev. 2003, 7, 349-361.

39. De Quervain, D.J.F.; Fischbacher, U.; Treyer, V.; Schellhammer, M.; Schnyder, U.; Buck, A.; Fehr, E. The neural basis of altruistic punishment. Science 2004, 305, 1254-1258.

40. Barrett, L.F.; Mesquita, B.; Ochsner, K.N.; Gross, J.J. The experience of emotion. Annu. Rev. Psychol. 2007, 58, 373-403. 
41. Russell, J.A. Core affect and the psychological construction of emotion. Psychol. Rev. 2003, 110, 145-172.

42. Böhm, G.; Pfister, H.-R. Action tendencies and characteristics of environmental risks. Acta Psychol. 2000, 104, 317-337.

43. Frijda, N.H. Emotion, cognitive structure, and action tendency. Cogn. Emot. 1987, 1, 115-143.

44. Zeelenberg, M.; Pieters, R. Feeling is for doing: A pragmatic approach to the study of emotions in economic behavior. In Social Psychology and Economics; DeCremer, D., Zeelenberg, M., Murnighan, J.K., Eds.; Erlbaum: Mahwah, NJ, USA, 2006; pp. 117-137.

45. Frank, R.H. Passions within Reason: The Strategic Role of the Emotions; Norton: New York, NY, USA, 1988.

46. Tangney, J.P.; Stuewig, J.; Mashek, D.J. Moral emotions and moral behavior. Annu. Rev. Psychol. 2007, 58, 345-372.

47. Geanakoplos, J.; Pearce, D.; Stacchetti, E. Psychological games and sequential rationality. Games Econ. Behav. 1989, 1, 60-79.

48. Böhm, G.; Brun, W. Intuition and affect in risk perception and decision making. Judgm. Decis. Mak. 2008, 3, 1-4.

49. Naqvi, N.; Shiv, B.; Bechara, A. The role of emotion in decision making: A cognitive neuroscience perspective. Curr. Dir. Psychol. Sci. 2006, 15, 260-264.

50. Mellers, B.A.; McGraw, A.P. Anticipated emotions as guides to choice. Curr. Dir. Psychol. Sci. 2001, 10, 210-214.

51. Slovic, P.; Peters, E. Risk perception and affect. Curr. Dir. Psychol. Sci. 2006, 15, 322-325.

52. Kirchsteiger, G.; Rigotti, L.; Rustichini, A. Your morals might be your moods. J. Econ. Behav. Organ. 2006, 59, 155-172.

53. Van Kleef, G.A.; Van Dijk, E.; Steinel, W.; Harinck, F.; van Beest, I. Anger in social conflict: Cross-situational comparisons and suggestions for the future. Group Decis. Negot. 2008, 17, $13-30$.

54. Reuben, E.; van Winden, F. Social ties and coordination on negative reciprocity: The role of affect. J. Public Econ. 2008, 92, 34-53.

55. Smith, R.H.; Kim, S.H. Comprehending envy. Psychological Bulletin 2007, 133, 46-64.

56. Schaubroeck, J.; Lam, S.S.K. Comparing lots before and after: Promotion rejectees' invidious reactions to promotees. Organ. Behav. Hum. Decis. Process. 2004, 94, 33-47.

57. Parks, C.D.; Rumble, A.C.; Posey, D.C. The effects of envy on reciprocation in a social dilemma. Personal. Soc. Psychol. Bull. 2002, 28, 509-520.

58. Zizzo, D.J.; Oswald, A. Are people willing to pay to reduce others' incomes? Working Paper. University of Warwick, Coventry, UK, 2001; pp. 39-65.

59. Gross, J.J.; Thompson, R.A. Emotion regulation: Conceptual foundations. In Handbook of Emotion Regulation; Gross, J.J., Ed.; Guilford Press: New York, NY, USA, 2007; pp. 3-24.

60. Güth, W.; van Damme, E. Information, strategic behavior, and fairness in ultimatum bargaining: An experimental study. J. Math. Psychol. 1998, 42, 227-247.

61. Knez, M.J.; Camerer, C. Outside options and social comparison in three-player ultimatum game experiments. Games Econ. Behav. 1995, 10, 65-94. 
62. Hertwig, R.; Ortmann, A. Experimental practices in economics: A methodological challenge for psychologists? Behav. Brain Sci. 2001, 24, 383-451.

63. Locey, M.L.; Jones, B.A.; Rachlin, H. Real and hypothetical rewards in self-control and social discounting. Judgm. Decis. Mak. 2011, 6, 552-564.

64. Kühberger, A.; Schulte-Mecklenbeck, M.; Perner, J. Framing decisions: Hypothetical and real. Organ. Behav. Hum. Decis. Process. 2002, 89, 1162-1175.

65. Pinheiro, J.C.; Bates, D.M. Mixed-Effects Models in S And S-Plus. Springer: New York, NY, USA, 2000.

66. Bakeman, R. Recommended effect size statistics for repeated measures designs. Behav. Res. Methods 2005, 37, 379-384.

67. Hertwig, R.; Ortmann, A. Deception in social psychological experiments: Two misconceptions and a research agenda. Soc. Psychol. Q. 2008, 71, 222-227.

68. Bonetti, S. Experimental economics and deception. J. Econ. Psychol. 1998, 19, 377-395.

69. Davis, D.D.; Holt, C.A. Experimental Economics; Princeton University Press: Princeton, NJ, USA, 1992.

70. Hertwig, R.; Ortmann, A. Deception in experiments: Revisiting the arguments in its defense. Ethics Behav. 2008, 18, 59-92.

71. Bröder, A. Deception can be acceptable. Am. Psychol. 1998, 53, 805-806.

72. Sieber, J.E.; Iannuzzo, R.; Rodriguez, B. Deception methods in psychology: Have they changed in 23 years? Ethics Behav. 1995, 5, 67-85.

73. Baron, R.M.; Kenny, D.A. The moderator-mediator variable distinction in social psychological research: Conceptual, strategic and statistical considerations. J. Personal. Soc. Psychol. 1986, 51, 1173-1182.

74. Bardsley, N. Dictator game giving: Altruism or artefact? Exp. Econ. 2008, 11, 122-133.

75. Colman, A.M. Cooperation, psychological game theory, and limitations of rationality in social interaction. Behav. Brain Sci. 2003, 26, 139-198.

76. Biel, A.; Thogersen, J. Activation of social norms in social dilemmas: A review of the evidence and reflections on the implications for environmental behavior. J. Econ. Psychol. 2007, 28, 93-112.

77. Fehr, E.; Rockenbach, B. Human altruism: Economic, neural, and evolutionary perspectives. Curr. Opin. Neurobiol. 2004, 14, 784-790.

78. Adams, J.S. Inequity in social exchange. In Advances in Experimental Social Psychology; Berkowitz, L., Ed.; Academic Press: New York, NY, USA, 1965; volume 2, pp. 267-299.

79. Van den Bos, K.; Peters, S.L.; Bobocel, D.R.; Ybema, J.F. On preferences and doing the right thing: Satisfaction with advantageous inequity when cognitive processing is limited. J. Exp. Soc. Psychol. 2006, 42, 273-289.

80. Tamir, M. What do people want to feel and why? Pleasure and utility in emotion regulation. Curr. Dir. Psychol. Sci. 2009, 18, 101-105.

81. Hey, J.D. Experimental economics and deception. A comment. J. Econ. Psychol. 1998, 19, 397-401.

82. Bardsley, N.; Cubitt, R.; Loomes, G.; Moffatt, P.; Starmer, C.; Sugden, R. Experimental Economics. Rethinking the Rules; Princeton University Press: Princeton, NJ, USA, 2010. 
83. Milgram, S. Behavioral study of obedience. J. Abnorm. Soc. Psychol. 1963, 67, 371-378.

84. Kahneman, D.; Tversky, A. Prospect theory: An analysis of decision under risk. Econometrica 1979, 47, 263-291.

85. Gray, E.K.; Watson, D. Assessing positive and negative affect via self-report. In Handbook of Emotion Elicitation and Assessment; Coan, J.A., Allen, J.J.B., Eds.; Oxford University Press: Oxford, UK, 2007; pp. 171-183.

86. Mauss, I.B.; Levenson, R.W.; McCarter, L.; Wilhelm, F.H.; Gross, J.J. The tie that binds? Coherence among emotion experience, behavior, and physiology. Emotion 2005, 5, 175-190.

87. Van Lange, P.A.M. The pursuit of joint outcomes and equality in outcomes: An integrative model of social value orientation. J. Personal. Soc. Psychol. 1999, 77, 337-349.

88. Henrich, J.; Heine, S.J.; Norenzayan, A. The weirdest people in the world? Behav. Brain Sci. 2010, 33, 61-135.

89. Trautmann, S.T. A tractable model of process fairness under risk. J. Econ. Psychol. 2009, 30, 809-813.

90. Oechssler, J.; Roider, A.; Schmitz, P.W. Cooling-Off in Negotiations-Does It Work? Center for Economic Policy Research: London, UK, 2006.

91. Bosman, R.; Sonnemans, J.; Zeelenberg, M. Emotions, Rejections, and Cooling off in the Ultimatum Game; Working Paper; University of Amsterdam, Amsterdam, The Netherlands, 2001.

(C) 2012 by the authors; licensee MDPI, Basel, Switzerland. This article is an open access article distributed under the terms and conditions of the Creative Commons Attribution license (http://creativecommons.org/licenses/by/3.0/). 